\title{
OA AND EXERCISE: THE IL-10 LINK
}

Patients with osteoarthritis (OA) are known to benefit from regular moderate exercise and a new study published in Arthritis Research \& Therapy suggests that an increase in the concentration of the anti-inflammatory cytokine interleukin (IL)-10 might be behind some of this beneficial effect.

Various cartilage matrix proteins can be used as markers to diagnose and assess the prognosis and treatment of $\mathrm{OA}$, but they are usually analyzed in blood or urine samples, sampling sites that are distant from the joint. "Samples of joint fluid and synovial tissue may more accurately represent local joint changes connected to the disease," says Ida Carøe Helmark, one of the key investigators on this study.

Employing a novel microdialysis method that uses intra-articular and perisynovial catheters, Helmark and colleagues were able to measure the concentration of a variety of inflammatory and cartilage markers within the joint itself. They used this method to obtain marker profiles from 29 white women with symptomatic knee OA who either completed a resistance exercise protocol $(n=16)$ or did not $(n=13)$.

The investigators found that intraarticular and perisynovial concentrations of IL-10 increased over the 3-hour postexercise period in the exercise group but remained unchanged over time in the nonexercise group. IL-10 is known to have a chondroprotective effect by inhibiting the release of inflammatory proteins by macrophages and activating synoviocytes and chondrocytes. "This would argue in favor of stimulating the joints of patients with early $\mathrm{OA}$, or patients at high risk of developing $\mathrm{OA}$, with exercise, as this would result in a release of IL-10, a response that potentially could have beneficial effect upon the control of the immune response," concludes Helmark.

Shreeya Nanda

Original article Helmark, I. C. et al. Exercise increases interleukin-10 levels both intraarticularly and peri-synovially in patients with knee osteoarthritis: a randomized controlled trial. Arthritis Res. Ther. 12, R126 (2010) 